\title{
\%
}

Joaquín López Vallés*

Juan M. Contreras Delgado de Cos**

\section{LAS AYUDAS PÚBLICAS EN LA CRISIS DE LA COVID-19}

Este trabajo resume los principales aspectos del Marco Temporal de ayudas para hacer frente a la COVID-19 aprobado por la Comisión Europea (CE), así como las medidas de ayuda notificadas por España que han sido aprobadas por la CE hasta la fecha. Se explican las diferencias entre Estados miembros de la Unión Europea y se ofrece una panorámica de los fondos europeos para la recuperación, destacándose la oportunidad que suponen tanto para conseguir los objetivos de digitalización y sostenibilidad medioambiental como para equilibrar, en su caso, las diferencias señaladas. Por último, se desarrolla la relación que cabe esperar de una política pública horizontal como la de competencia para favorecer el objetivo de la sostenibilidad.

Palabras clave: ayudas públicas, COVID-19, fondos europeos de recuperación, política de competencia, sostenibilidad.

Clasificación JEL: F35, H12, H25.

\section{Introducción}

La pandemia de la COVID-19, que ha provocado una crisis sanitaria mundial sin precedentes, ha derivado también en una crisis económica y social sin parangón en la historia reciente. Del conjunto de instrumentos de política pública de los que disponen los poderes públicos para hacer frente a sus consecuencias, la concesión de ayudas o subvenciones es, sin duda, uno de los más relevantes.

\footnotetext{
* Director del Departamento de Promoción de la Competencia de la CNMC.

** Subdirector de Ayudas Públicas e Informes de Proyectos Normativos de la CNMC.

Versión de marzo de 2021.

DOI: https:/doi.org/10.32796/bice.2021.3134.7181
}

Así lo ha entendido la propia Comunidad Europea (en adelante, CE), que, como ya sucediera en la crisis financiera y económica de 2008, ha aprobado diferentes medidas (Marco Temporal) que pretenden adaptar la normativa vigente de ayudas de Estado de la Unión Europea (UE) a la situación excepcional de la pandemia.

Los Estados miembros (EE MM) de la UE han venido utilizando con gran intensidad la flexibilidad ofrecida por el Marco Temporal para canalizar ayudas a los operadores presentes en sus territorios con gran rapidez. Ello ha permitido mitigar los efectos nocivos de la crisis sobre la actividad de las empresas y los trabajadores. Así lo ha realizado también nuestro país, que ha desplegado diferentes instrumentos de $\square$ 
ayuda que han sido objeto de notificación a la CE para su preceptiva autorización.

Uno de los riesgos de la crisis, a tenor de las diferentes capacidades financieras de los EE MM y de la política expansiva de ayudas públicas, es la creación de diferencias importantes entre las empresas asentadas en cada Estado, lo que acrecienta el riesgo de crear importantes desequilibrios en el mercado interior.

En este sentido, debe destacarse el importante papel que deben jugar los «fondos europeos». El Consejo Europeo de 21 julio de 2020 aprobó un paquete de medidas que incluía un Marco Financiero Plurianual (MFP) reforzado para el periodo 2021-2027 y la puesta en marcha de un Instrumento Europeo de Recuperación (Next Generation EU), que supone el mayor paquete de estímulo jamás financiado a través del presupuesto de la UE, con un total de 1,8 billones de euros.

La gestión eficiente de estos recursos es una obligación. De lo contrario, será muy difícil la consecución de los objetivos de transición digital y sostenibilidad medioambiental. En este artículo nos centramos en analizar la relación entre la política de competencia y el objetivo de sostenibilidad. Cabe recordar que la política de competencia no tiene entre sus objetivos primordiales la sostenibilidad, sino la eficiencia y el buen funcionamiento de los mercados, en beneficio de los consumidores. Sin embargo, la relación entre las normas de competencia y la sostenibilidad, no solo desde el punto de vista medioambiental, sino también en sus dimensiones económica y social, es una de las cuestiones más relevantes para la agenda de las autoridades de competencia en los próximos años.

\section{La flexibilidad de los controles europeos: el Marco Temporal}

Desde el inicio de la crisis sanitaria provocada por la COVID-19, la CE ha aprobado diferentes medidas de carácter temporal que pretenden flexibilizar la normativa vigente de ayudas de Estado de la UE debido a la situación excepcional que atravesamos como consecuencia de la pandemia, como ya sucediera en la crisis financiera y económica iniciada en 2008.

No se trata de instrumentos que modifiquen las normas recogidas en los tratados fundacionales de la Unión Europea (en especial los artículos 107-109 del Tratado de Funcionamiento de la UE, en adelante TFUE), ni tampoco, en líneas generales, las precisadas en derecho derivado, entre otros motivos, porque no tienen naturaleza normativa.

Sin embargo, al igual que sucedió en la crisis anterior reseñada, estas medidas presentan una relevancia práctica evidente, por cuanto delimitan las principales vías de concesión de ayudas de Estado con las que cuentan los Estados miembros para hacer frente a las consecuencias sanitarias, sociales y económicas de la pandemia.

El proceso se inició el pasado 13 de marzo de 2020, cuando la CE publicó la «Comunicación de Respuesta Coordinada frente al brote de COVID-19 en la UE». En ella se desgranaban diferentes aspectos relacionados con la respuesta europea al estallido de la pandemia, uno de los cuales tenía que ver con la concesión de ayudas públicas.

Pero, sin duda, el hito fundamental se produjo el 19 de marzo de 2020, con la publicación por la CE de la Comunicación «Marco Temporal relativo a las medidas de ayuda estatal destinadas a respaldar la economía en el $\triangleright$ 
contexto del actual brote de COVID-19» (en adelante, Marco Temporal). Esta comunicación se ha modificado hasta en cinco ocasiones ${ }^{1}$ (la última en enero de 2021). En líneas generales, las modificaciones han ido configurando un Marco Temporal que, si bien inicialmente se centraba casi exclusivamente en ofrecer soluciones para afrontar los retos de salud pública y de impacto social, se ha ido ampliando hacia instrumentos más relacionados con la recuperación económica.

A pesar de que el Marco Temporal es el instrumento fundamental para vehicular las ayudas de Estado frente a la crisis, en paralelo se han producido otras iniciativas en materia de ayudas de Estado, como la realización de ajustes sobre la normativa de ayudas exentas de notificación y ayudas de minimis, la prórroga de las Directrices UE sobre ayudas de naturaleza sectorial o un paquete de medidas de apoyo al sector del transporte y turismo, entre las que destaca la recomendación de 13 de mayo de 2020 relativa a los bonos ofrecidos a los pasajeros y a los viajeros como alternativa al reembolso de viajes combinados y servicios de transporte.

El Marco Temporal, cuya vigencia está fijada actualmente hasta el 31 de diciembre de 2021, ofrece unas directrices sobre la compatibilidad de las ayudas con el artículo 107.3.b TFUE ${ }^{2}$.

\footnotetext{
1 El 3 de abril de 2020 se produce la primera modificación de la Comunicación por la que se modifica el Marco Temporal, ampliando cinco tipos adicionales de medidas, relacionadas con el apoyo a líneas de investigación y ensayos, ayudas por costes laborales y de seguridad social. Posteriormente, el 8 de mayo fue publicada por parte de la CE una segunda modificación del Marco Temporal, que amplía las posibilidades de intervención pública a recapitalizaciones y a la compra de deuda subordinada en condiciones favorables. Posteriormente, el 29 de junio se produjo la tercera modificación del Marco Temporal; el 13 de octubre, la cuarta, y el 28 de enero de 2021 se aprobó la quinta modificación.

2 Art. 107.3 b TFUE: «Podrán ser consideradas compatibles con el mercado interior: [...] b) las ayudas para fomentar la realización de un proyecto importante de interés común europeo o destinadas a poner remedio a una grave perturbación en la economía de un Estado miembro».
}

Ello sobre la base de que el brote de COVID-19, dada su afectación generalizada a toda la UE, constituye una «grave perturbación en la economía de los Estados miembros». Todas las ayudas concedidas bajo este Marco deben ser objeto de notificación previa a la CE para su correspondiente autorización.

El Marco Temporal ha sido el principal instrumento para permitir que se acelerase la aprobación de las diferentes medidas de ayudas diseñadas por los Estados miembros ${ }^{3}$. Su objetivo es hacer frente a las dificultades que están encontrando las empresas, al tiempo que mantener la integridad del mercado interior de la UE y la igualdad de condiciones.

Todo ello durante un periodo de tiempo limitado, para poner remedio a la escasez de liquidez a la que se enfrentan las empresas y garantizar que las perturbaciones ocasionadas por la pandemia no socaven su viabilidad, especialmente la de las pymes. De particular interés es la condicionalidad recogida en el Marco acerca de que la ayuda concedida no podrá estar condicionada al traslado de una actividad de producción o de otra actividad del beneficiario desde otro país perteneciente al espacio económico europeo (EEE) al territorio del Estado miembro que concede la ayuda. De lo contrario, se podrían haber creado incluso mayores desequilibrios entre EE MM.

Otro tema de singular relevancia es la exigencia de solvencia de las entidades $\triangleright$

3 Además del Marco Temporal, el TFUE ofrece otras vías para amparar las ayudas públicas en la situación actual. Por ejemplo, sobre la base del artículo 107, apartado 3, letra c, del TFUE, y tal como se especifica en las directrices sobre ayudas estatales de salvamento y de reestructuración, los Estados miembros pueden notificar a la Comisión regímenes de ayuda para satisfacer necesidades de liquidez agudas y apoyar a las empresas que se enfrentan a dificultades financieras, también debidas al brote de COVID-19 o agravadas por él. Además, sobre la base del artículo 107, apartado 2, letra b, del TFUE, los Estados miembros también pueden indemnizar a las empresas de los sectores especialmente afectados por el brote (por ejemplo, el transporte, el turismo, la cultura, la hostelería y el comercio minorista) y/o los organizadores de eventos cancelados por los daños y perjuicios sufridos como consecuencia de brote y provocados directamente por él. 
receptoras. Los beneficiarios no pueden encontrarse en una situación de crisis antes del 31 de diciembre de $2019^{4}$. Además, la ayuda debe concederse antes del 31 de diciembre de 2021.

Existe una variada tipología de ayudas incluidas en la comunicación que son consideradas compatibles con el mercado interior:

- Importes limitados y temporales de ayuda a empresas que se enfrentan a una súbita escasez o, incluso, a una falta de liquidez. La ayuda puede concederse en forma de subvenciones directas, ventajas fiscales y de pago, anticipos reembolsables, garantías, préstamos y capital, con el límite global de 1,8 millones de euros por empresa ${ }^{5}$.

- Garantías de préstamos. Deben estar limitadas a un máximo de seis años y no pueden superar el $90 \%$ del principal del préstamo cuando las pérdidas se imputen proporcionalmente y en condiciones idénticas a la entidad de crédito y al Estado (y el $35 \%$ si las pérdidas se imputan primero al Estado). Las primas deben respetar unos importes mínimos.

- Bonificación de los tipos de interés de los préstamos. Siempre que el tipo de interés sea, al menos, equivalente al tipo base (IBOR a un año o equivalente) más una prima mínima6.

\footnotetext{
4 Como excepción a lo anterior, se podrán conceder ayudas a microempresas o pequeñas empresas (en el sentido del Anexo I del Reglamento General de Exención por Categorías) que ya estuvieran en crisis el 31 de diciembre de 2019, siempre y cuando no se encuentren inmersas en un procedimiento concursal con arreglo a su derecho nacional y no hayan recibido una ayuda de salvamento o de reestructuración.

5 Todas las cifras utilizadas constituyen importes brutos, es decir, antes de impuestos y otras retenciones. Para ciertos sectores, existen umbrales diferentes, como los 270.000 euros por empresa activa en los sectores de la pesca y la acuicultura o los 225.000 euros por empresa activa en la producción primaria de productos agrícolas.

6 Las primas son las mismas que las previstas para los avales.
}

- Garantías y préstamos canalizados a través de entidades de crédito $u$ otras entidades financieras. Estas entidades deben, en la medida de lo posible, repercutir las ventajas de la garantía pública o de las bonificaciones de los tipos de interés de préstamos a los beneficiarios finales. Por ejemplo, en forma de mayores volúmenes de financiación o menores requisitos en materia de garantías.

- Seguro de créditos a la exportación a corto plazo. Se permite su utilización para la exportación a cualquier país.

- Ayudas a la investigación y desarrollo relacionadas con la COVID-19. En forma de subvenciones directas, anticipos reembolsables o beneficios fiscales a la I+D en materia de coronavirus y otras medidas antivirales pertinentes.

- Ayudas a la inversión destinada a las infraestructuras de ensayo y ampliación de escala. Se permiten subvenciones directas, anticipos reembolsables o ventajas fiscales con el fin de apoyar las inversiones que permitan la construcción o mejora de las infraestructuras necesarias para desarrollar y ensayar productos útiles para hacer frente al brote de coronavirus hasta su primer despliegue industrial?.

- Ayudas a la inversión para la producción de productos relacionados con la COVID-19. Se permiten subvenciones directas, anticipos reembolsables y ventajas fiscales para apoyar las inversiones que permitan la rápida fabricación de productos que sirvan para combatir el brote.

\footnotetext{
7 Entre ellos, se cuentan productos como medicamentos (incluidas las vacunas) y tratamientos; aparatos y dispositivos médicos (incluidos respiradores y ropa de protección, así como herramientas de diagnóstico); desinfectantes; herramientas de recogida y procesamiento de datos útiles para luchar contra la propagación del virus.
} 
- Aplazamientos del pago de impuestoso cotizaciones a la Seguridad Social. Se podrán conceder aplazamientos específicos del pago de impuestos y de las cotizaciones a la Seguridad Social en los sectores, regiones o tipos de empresas más afectados por el brote. La fecha límite para el aplazamiento no podrá ser posterior al 31 de diciembre de 2022.

- Subsidios salariales para los empleados a fin de evitar las reducciones de plantilla durante el brote de COVID-19. Para mitigar el impacto de la crisis sobre los trabajadores, se podrá contribuir a los costes salariales de las empresas en sectores o regiones más afectadas por la pandemia. El subsidio salarial mensual no deberá superar el $80 \%$ del salario bruto mensual del personal beneficiario (o el $80 \%$ de los ingresos equivalentes al salario mensual medio de la persona que trabaja por cuenta propia).

- Medidas de recapitalización. Estas ayudas pueden realizarse mediante la suscripción de participaciones en el capital o mediante otros instrumentos híbridos de capital, como préstamos con derecho de participación en los beneficios, suscripción de participaciones sin derecho a voto, bonos convertibles o bonos no garantizados. Entre las condiciones que se debe cumplir por los Estados cabe destacar las siguientes:

- Elegibilidad. Debe acreditarse que: i) en ausencia de recapitalización, el beneficiario quebraría o entraría en serias dificultades para mantener sus operaciones; ii) existe un interés general en mantener a la empresa; iii) el beneficiario no puede financiarse en los mercados en términos razonables; $y$ iv) el beneficiario no estuviera ya en dificultades a 31 de diciembre de 2019, salvo las ayudas a microempresas o pequeñas empresas ya referidas.

- Importe necesario y proporcionado. La ayuda debe limitarse a la mínima imprescindible para asegurar la viabilidad de la empresa y no ir más allá de restaurar la estructura de capital del beneficiario antes del brote de coronavirus.

- Remuneración suficiente de la participación estatal. El Estado debe ser suficientemente remunerado por los riesgos que asume a través de la ayuda de recapitalización. Una remuneración elevada incentiva a los beneficiarios a recomprar la participación del Estado lo antes posible. En el caso de compra de acciones, el precio de compra por el Estado no debe superar el precio medio de la acción durante los quince días anteriores. En el caso de los instrumentos híbridos de capital, también se regula una remuneración mínima de la participación estatal y se debe incluir un mecanismo de step-up de la remuneración una vez se haya convertido el instrumento en capital.

- Estrategia de salida. Los beneficiarios que no sean pymes que hayan recibido una recapitalización de más del $25 \%$ de su capital deben tener una estrategia de salida del Estado en el plazo de doce meses desde la fecha de concesión de la ayuda (salvo que la participación del Estado se reduzca por debajo de ese nivel en ese plazo). Además, si la ayuda del Estado no se ha reducido por debajo del $15 \%$ del capital al cabo de seis años (siete $\triangleright$ 
años para empresas no cotizadas), deberá presentarse un plan de reestructuración de la empresa, que debe aprobarse por la Comisión.

- Gobernanza y competencia. Se establecen diferentes condiciones para evitar que la empresa beneficiaria mejore su posición competitiva respecto a la situación previa a recibir la ayuda, como límites a su capacidad de adquirir otras empresas ${ }^{8}$ y de utilizar los fondos para sus propias actividades ${ }^{9}$, a la remuneración de accionistas ${ }^{10} y$ directivos ${ }^{11}$, y a la publicidad de la participación pública ${ }^{12}$. Además, si la empresa receptora tiene poder de mercado significativo y la participación del Estado es superior a 250 millones de euros, deben instrumentarse remedios estructurales o de comportamiento para mantener la competencia efectiva.

- Transparencia pública y presentación de informes. Si la ayuda se otorga a los beneficiarios como parte de un esquema nacional, los EE MM deben publicar detalles sobre la identidad de las empresas que han recibido ayuda y la cuantía recibida dentro de los tres meses posteriores a la recapitalización. Además, los beneficiarios tienen que publicar información sobre el uso

\footnotetext{
8 Hasta que se canjee al menos el $75 \%$ de la recapitalización, las grandes empresas no pueden, en principio, adquirir una participación de más del $10 \%$ de competidores, proveedores ni clientes.

9 La recapitalización no puede utilizarse para apoyar las actividades económicas de empresas integradas que estaban en dificultades económicas antes del 31 de diciembre de 2019.

10 Hasta que el Estado haya salido por completo del capital, las empresas no pueden otorgar dividendos ni recomprar otras acciones que las del Estado.

11 Hasta que se haya canjeado al menos el $75 \%$ de la recapitalización, no pueden percibir remuneraciones superiores a su remuneración fija a diciembre de 2019 y no pueden percibir variables ni bonus.

12 La participación del Estado en el capital no puede utilizarse con fines comerciales.
}

de la ayuda recibida, en particular cómo respalda las actividades de la empresa en línea con las obligaciones nacionales y de la UE vinculadas a la transformación verde y digital.

- Ayudas por los costes fijos no cubiertos de aquellas empresas para las cuales el brote de COVID-19 hubiese acarreado la suspensión o reducción de su actividad empresarial (reducción de su volumen de negocios de, al menos, un $30 \%$ con respecto al mismo periodo de 2019), con el límite global de 10 millones de euros por empresa.

\section{Las ayudas públicas en España y los desequilibrios en el mercado interior en la UE}

\subsection{Las ayudas públicas concedidas en España para afrontar la pandemia}

Sobre la base de las amplias posibilidades que ofrece el Marco Temporal comentado, el Reino de España ha desplegado diferentes instrumentos de ayuda, que han sido objeto de notificación a la CE. Si bien inicialmente se optó por notificar instrumentos de ayuda más homogéneos, posteriormente se ha utilizado la vía de los instrumentos «paraguas» que abarcan diferentes posibilidades de ayuda.

Además, los esquemas de ayudas se han ido ampliando para adaptarse a las modificaciones efectuadas en el Marco Temporal de la CE. Pasamos a exponer las líneas de ayudas ${ }^{13} \square$

\footnotetext{
13 También se han concedido otras ayudas de diverso tenor (no vinculadas al Marco Temporal): apoyo a la internacionalización de las empresas. Se extiende a empresas cotizadas (ya no solo a pymes, por tanto) el acceso a la línea extraordinaria de garantías públicas de la Compañía Española de Seguro de Crédito a la Exportación (CESCE), aprobada mediante el Real Decreto-ley 8/2020; Refuerzo del Fondo de Inversiones en el Exterior (FIEX), gestionado por la Compañía Española
} 
notificadas por el Reino de España acogiéndose al Marco Temporal, siguiendo la secuencia temporal en que se han producido:

- Línea de avales a empresas y autónomos para paliar los efectos económicos de la COVID-1914. Tiene como objetivo garantizar que haya suficiente liquidez disponible en el mercado, contrarrestar el daño producido a las empresas afectadas por el brote y preservar la continuidad de la actividad económica. Estas garantías cubrirán nuevos préstamos y operaciones de refinanciamiento de autónomos y empresas. El Instituto de Crédito Oficial (ICO) será el administrador de la medida a través de entidades de crédito y otras entidades financieras.

- Régimen paraguas. Marco Nacional Temporal relativo a las medidas de ayuda a empresas y autónomos consistentes en subvenciones directas, anticipos reembolsables, ventajas fiscales, garantías de préstamos y bonificaciones de tipos de interés en préstamos destinadas a respaldar la economía ${ }^{15}$.

- Régimen paraguas. Segundo Marco Nacional Temporal para las ayudas estatales relacionadas con la contención del

de Financiación del Desarrollo (COFIDES), ampliando de 10 a 100 millones de euros la dotación de este fondo; puesta en marcha de los planes de apoyo al sector turístico: asegurar la protección de los deudores hipotecarios cuyo inmueble se encuentre afecto a una actividad hotelera, de alojamientos turísticos y agencias de viajes, a través del otorgamiento de un periodo de moratoria de hasta doce meses para las operaciones financieras suscritas entre los referidos deudores hipotecarios y las entidades de crédito, entre otras medidas; planes de automoción (250 millones de euros), estableciendo las bases precisas para la concesión de ayudas para la puesta en marcha del programa RENOVE de apoyo a la renovación del parque automovilístico. Se califican como ayudas de minimis a efectos del cumplimiento de la normativa de la UE en materia de ayudas de Estado.

14 SA.56803 SPAIN. Notificada a la CE y aprobada por ésta sin objeciones el 24 de marzo de 2020.

15 SA.56851(2020/N) SPAIN. Notificada a la CE y aprobada por ésta sin objeciones el 2 de abril de 2020. brote de COVID-19 ${ }^{16}$. Se añade la posibilidad de otorgar ayudas:

- Para la investigación y el desarrollo relevantes del coronavirus.

- A la inversión destinada a las infraestructuras de ensayo y ampliación de escala.

- A la inversión para la producción de productos relevantes para el coronavirus.

- En forma de aplazamientos del pago de impuestos y/o de cotizaciones a la Seguridad Social.

- En forma de subsidios salariales para los empleados a fin de evitar las reducciones de plantilla durante la pandemia de coronavirus.

- Fondo de apoyo a la solvencia de empresas estratégicas ${ }^{17}$. El Real Decreto-ley $25 / 2020$, de 3 de julio, de medidas urgentes para apoyar la reactivación económica y el empleo, contempla un variado conjunto de medidas, entre las que destaca la creación de un nuevo fondo, con una dotación global de 10.000 millones de euros, para dar apoyo financiero a las empresas no financieras estratégicas solventes que se hayan visto especialmente afectadas por la COVID-1918. El fondo, gestionado a través de la Sociedad Estatal de Participaciones Industriales (SEPI), está destinado exclusivamente a empresas consideradas estratégicas para el tejido productivo nacional o regional, entre $D$

16 SA.57019 (2020/N) SPAIN. Notificada a la CE y aprobada por ésta sin objeciones el 24/04/2020.

17 State Aid SA.57659(2020/N) SPAIN. COVID-19. Notificada a la CE y aprobada por ésta sin objeciones el 31 de julio de 2020.

18 Dicho fondo ha sido desarrollado por la Orden PCM/679/2020, de 23 de julio, por la que se publica el Acuerdo del Consejo de Ministros de 21 de julio de 2020, que establece el funcionamiento y las características del mismo. Las intervenciones superiores a 250 millones de euros por beneficiario serán objeto de notificación individualizada, al amparo de lo previsto en el Marco Temporal. 
otros motivos, por su sensible impacto social y económico, su relevancia para la seguridad, la salud de las personas, las infraestructuras, las comunicaciones, o su contribución al buen funcionamiento de los mercados. Se exige que sean empresas no financieras con domicilio social y principales centros de trabajo situados en España. Las operaciones financiadas con cargo al fondo tienen un importe mínimo de 25 millones de euros por beneficiario, salvo casos excepcionales debidamente justificados.

- Nueva línea de avales ${ }^{19}$. Entre las medidas previstas en el citado Real Decreto-ley 25/2020 destaca una nueva línea de avales ICO de 40.000 millones de euros, orientada principalmente a financiar inversiones productivas. Es continuista de las líneas de avales anteriormente existentes, si bien destaca el hecho de que, a diferencia de estas, que pretendían contrarrestar el daño producido a las empresas afectadas por el brote y las acuciantes necesidades de liquidez, la nueva línea tiene como objeto inversiones productivas.

- Prolongación del Marco Nacional Temporal y modificación de la línea de ava$l e s^{20}$. Se permite que la duración de estos avales se extienda hasta ocho años.

- Avales a empresas que han llegado a convenios con acreedores ${ }^{21}$. Se trata de un régimen de apoyo en forma de

\footnotetext{
19 SA.58096 (2020/N) Spain COVID-19: Amendments to SA.56803 (2020/N) Guarantee scheme. La CE aprobó el 5 de agosto de 2020 la modificación de la «línea de avales para las empresas y autónomos para paliar los efectos económicos del COVID-19» aprobada el 24 de marzo (State Aid SA.56803)

20 SA.58778 COVID-19: Amendments to SA.56851 (2020/N) Conditions of the guarantees on loans and prolongation. La CE la aprobó sin objeciones el 2 de octubre de 2020.

21 SA.59045 (2020/N) SPAIN. Fue notificada a la CE y aprobada por ésta sin objeciones el 23 de noviembre de 2020.
}

garantías para acceder a financiación, ofrecidas a las empresas que han sufrido daños sustanciales debido al brote de COVID-19 y que, por encontrarse en un procedimiento de insolvencia y haber llegado a un acuerdo de acreedores que están cumpliendo, no han podido adherirse a otros programas.

- Mecanismo de reaseguro de crédito comercial $^{22}$. La medida habilita la creación de un mecanismo de reaseguro por parte del Consorcio de Compensación de Seguros a créditos comerciales concedidos. El mecanismo está dotado con un fondo de 500 millones de euros.

- Medidas para reforzar la solvencia de autónomos y empresas. El Real Decreto-ley 5/2021, de 12 de marzo, de medidas extraordinarias de apoyo a la solvencia empresarial en respuesta a la pandemia de la COVID-19, ha aprobado tres líneas de ayudas destinadas a empresas viables en los sectores más afectados por la pandemia.

- Línea de 7.000 millones de euros para ayudas directas a autónomos y empresas. Podrán beneficiarse autónomos y empresas viables que hayan tenido una caída de sus ingresos de, al menos, el $30 \%$ en 2020 respecto al año anterior. Las ayudas van en un rango de entre 3.000 y 200.000 euros por beneficiario. Estas ayudas se gestionarán por las comunidades autónomas, destacándose dos condiciones:

- Domicilio fiscal: solo se pueden conceder ayudas a empresas con sede fiscal en el territorio de la comunidad, $D$

22 SA.58458 SPAIN. Notificada a la CE y aprobada por ésta sin objeciones el 4 de diciembre de 2020 . 
CUADRO 1

IMPORTE TOTAL DE LAS AYUDAS APROBADAS EN ESPAÑA

\begin{tabular}{|c|c|c|c|c|c|}
\hline Tipos de medidas & $\begin{array}{l}\text { Importe anunciado } \\
\text { (millones de euros) }\end{array}$ & $\begin{array}{c}\% \text { sobre } \\
\text { el PIB } 2020\end{array}$ & $\begin{array}{l}\text { Importe utilizado } \\
\text { (millones de euros) }\end{array}$ & $\begin{array}{l}\% \text { sobre el imp. } \\
\text { anunciado }\end{array}$ & $\begin{array}{l}\% \text { sobre el } \\
\text { PIB } 2020\end{array}$ \\
\hline 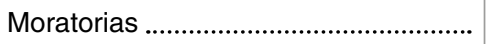 & - & - & 54.043 & - & 4,9 \\
\hline  & 145.060 & 13,1 & 91.314 & 62,9 & 8,2 \\
\hline Participación accionarial ........................ & 10.000 & 0,9 & 475 & 4,8 & 0,0 \\
\hline 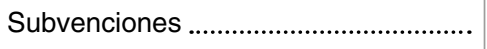 & 26.846 & 2,4 & 20.951 & 78,04 & 1,9 \\
\hline 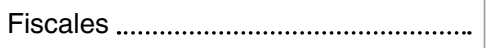 & 14.463 & 1,3 & 8.829 & 61,0 & 0,8 \\
\hline Otras medidas de gasto (est.) .............. & 19.595 & 1,8 & 12.130 & 61,9 & 1,1 \\
\hline 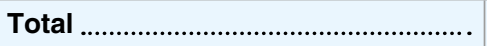 & 215.965 & 19,5 & 187.742 & 61,9 & 16,9 \\
\hline
\end{tabular}

salvo en el caso de empresas de capital social superior a 10 millones de euros, a las que bastará acreditar que cuentan con una actividad en ese territorio.

- Sectores incluidos: son muy diversos, desde la minería del hierro hasta sectores industriales (agroalimentario, textil, editorial, minerales, joyería, óptica...) y toda clase de actividades de comercio o turismo.

- Ayudas para la reestructuración de deuda financiera COVID: incluye un conjunto de medidas de apoyo y flexibilización de los préstamos que cuenten con aval público, permitiendo así que el ICO se incorpore a los procesos de refinanciación y reestructuración que pacten los bancos y sus clientes, protegiendo también la estabilidad financiera. Este eje se ve apoyado mediante la creación de una nueva línea para la reestructuración de deuda financiera con aval del Estado, dotada con 3.000 millones de euros.

- Fondo de recapitalización de empresas: dirigido a reforzar los balances de empresas que eran viables en diciembre de 2019 pero que se enfrentan a problemas de solvencia por la pandemia. Este fondo está dotado con 1.000 millones de euros y será gestionado por COFIDES.

El Ministerio de Asuntos Económicos y Transformación Digital ofrece mayor detalle en su web sobre las ayudas concedidas en España durante los meses de pandemia ${ }^{23}$. Según el citado ministerio, en nuestro país se han concedido más de 215.965 millones de euros en ayudas desde el inicio de la pandemia, y cuyo desglose se puede ver en el Cuadro 1.

\subsection{Los posibles desequilibrios en el mercado interior}

Las ayudas públicas constituyen uno de los mecanismos más eficaces para resolver determinados fallos del mercado (principalmente, externalidades, bienes públicos e información asimétrica) que dificultan que éste alcance asignaciones óptimas.

Sin embargo, las ayudas públicas también pueden distorsionar la competencia en los mercados y el funcionamiento del mercado interior en la UE. El control de las ayudas de Estado es consustancial al proceso de integración de $\triangle$

23 https://portal.mineco.gob.es/es-es/ministerio/covid19/Paginas/ ART_CABECERA.aspx. 
mercados de la UE. En ausencia de este control, sería difícil evitar que los países entraran en una carrera por apoyar financieramente a sus empresas, distorsionando el libre juego competitivo y las ventajas de la integración comercial europea.

Por ello, las ayudas públicas están sujetas al control de las ayudas de Estado previsto en los tratados fundacionales y el derecho derivado. Este control se fundamenta en la sujeción a unos objetivos específicos que se consideran compatibles con el mercado interior y en un análisis de necesidad y proporcionalidad, bajo el que se evalúan y confrontan los efectos positivos con los negativos de las ayudas estatales y solamente se admiten cuando dominan los primeros sobre los segundos.

La crisis experimentada a raíz de la pandemia es de tal entidad que puede terminar expulsando del mercado a empresas que eran perfectamente viables antes de la crisis. En este contexto singular, las ayudas de Estado juegan un papel fundamental para contribuir a proveer de liquidez y mejorar la capacidad financiera, inversora y productiva de las empresas. Es por ello que resulta vital la flexibilidad ofrecida en el ámbito del control de las ayudas de Estado por el Marco Temporal para canalizar rápidamente importantes volúmenes de ayuda a las empresas. En octubre de 2020, la Comisión Europea cifró en casi tres billones de euros el volumen de apoyo en forma de ayudas de Estado autorizadas hasta ese momento en el conjunto de la UE ${ }^{24}$.

Ahora bien, la mayor flexibilidad ofrecida por el Marco Temporal también puede llevar a que se generen notables diferencias en la capacidad competitiva de las empresas europeas, en

24 https://ec.europa.eu/commission/presscorner/detail/en/ STATEMENT_20_1805. función de la capacidad de apoyo de los Estados miembros donde estén domiciliadas.

La CE ha sido consciente de este riesgo, pero no ha considerado conveniente introducir, por ejemplo, medidas limitativas por EE MM que pudieran equilibrar las diferencias potenciales. Sin embargo, se han establecido otras medidas para mitigarlo, como la sujeción a ciertos umbrales máximos de ayuda a las empresas o la obligatoriedad de cumplir ciertos requisitos de comportamiento empresarial para preservar la competencia efectiva. Pero, sin duda, como veremos en el apartado siguiente, el principal mecanismo de compensación reside en la recepción por los EE MM de fondos europeos y la sujeción de los mismos a ciertos objetivos de transición digital y medioambiental.

En el presente apartado se realiza una comparativa de los niveles de ayudas públicas otorgados por los EE MM a partir de la información disponible. Debe señalarse que existen determinados factores que dificultan una adecuada comparativa entre EE MM en cuanto a volúmenes de ayuda.

- En primer lugar, cuando se notifica un esquema de ayudas (y no una ayuda a una empresa individual), no es obligatorio indicar el importe total de las ayudas del esquema, por lo que en algunos casos se desconoce la cuantía global hasta cierto tiempo después (en algunos casos, muchos meses después).

- En segundo lugar, la reducida información proporcionada por la CE hasta la fecha respecto al total de ayudas notificadas por país, de forma que no será probablemente hasta bien entrado 2021 cuando se publicarán los primeros avances de dicha concesión con una suficiente desagregación nacional. 
CUADRO 2

IMPORTES CONSIGNADOS EN LAS DECISIONES DE AYUDAS DE ESTADO AUTORIZADAS POR LA CE

(Junio de 2020, septiembre de 2020 para España)

\begin{tabular}{|c|c|c|c|}
\hline Estado miembro de la UE & $\begin{array}{l}\text { Cantidad nominal } \\
\text { (millones de euros) }\end{array}$ & $\begin{array}{l}\text { Cantidad utilizada } \\
\text { (millones de euros) }\end{array}$ & $\begin{array}{l}\text { \% sobre PIB nacional } \\
\text { (cantidad utilizada) }\end{array}$ \\
\hline 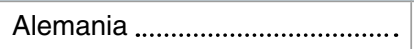 & 820.000 & 33.500 & 1 \\
\hline Italia & 450.000 & 51.200 & 3 \\
\hline 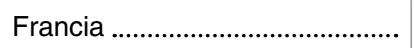 & 300.000 & 107.000 & 4 \\
\hline España & 100.000 & 52.700 & 4 \\
\hline 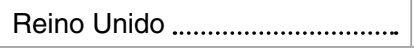 & 380.000 & 47.000 & 2 \\
\hline
\end{tabular}

- En tercer lugar, el hecho de que, incluso en los casos en los que se identifica el importe global de un esquema, una vez aprobado, no exista una obligación de que sean utilizados en su totalidad los fondos públicos comprometidos.

Partiendo de estas limitaciones, el Cuadro 2 compara los niveles de ayudas vinculadas a la pandemia ${ }^{25}$ autorizados por la UE (cantidad nominal) y desembolsados (cantidad utiliza$\mathrm{da}^{26}$ ) de los principales EE MM (incluyendo a Reino Unido, que, a la fecha de la comparativa, formaba parte de la UE) en junio de 2020.

Del Cuadro 2 se desprende que Alemania se sitúa como líder destacado en lo referente al volumen de ayuda aprobada. Sin embargo, el desembolso efectivo por los EE MM de la ayuda nominalmente aprobada por la CE había sido, en los primeros meses de la crisis, relativamente bajo. España encabezaba el ranking de los países que más cantidad de la ayuda aprobada habían desembolsado, junto con Francia. En términos del producto interior bruto (PIB), las ayudas desembolsadas por España

25 Ya sea por decisiones vinculadas al Marco Temporal o a otro tipo de artículos habilitantes del TFUE.

26 El dato se refiere a ayudas en forma de avales, si bien este dato puede aproximarse al del total de ayudas concedidas, pues esta ha sido la forma preferente de gestionar los programas de ayudas, particularmente cuando sus cantidades son elevadas. y Francia correspondían al $4 \%$ de sus PIB respectivos ${ }^{27}$.

\section{Un análisis prospectivo de los fondos europeos: el reto de la sostenibilidad}

\subsection{Los fondos europeos de recuperación}

El Consejo Europeo de 21 julio de 2020 aprobó un paquete de medidas que incluía un Marco Financiero Plurianual (MFP) reforzado para el periodo 2021-2027 y la puesta en marcha de un Instrumento Europeo de Recuperación (Next Generation EU). Suponen el mayor paquete de estímulo jamás financiado a través del presupuesto de la UE, con un total de 1,8 billones de euros.

El Instrumento Europeo de Recuperación (Next Generation EU) es un instrumento temporal dotado con 750.000 millones de euros. Se distribuye en los siguientes mecanismos.

Por un lado, el elemento central del sistema es el Mecanismo Europeo de Recuperación $\square$

27 En referencias de prensa (REUTER, 16 de marzo de 2021), citando fuentes de la $\mathrm{CE}$, se han recogido aproximaciones realizadas ya para finales de 2020 en los que sería Francia el principal concedente de ayudas (155.360 millones de euros), seguido de Italia (107.900 millones euros), Alemania (104.250 millones euros) y España (90.800 millones euros). En términos de ayuda en relación al PIB, España estaría en cabeza $(7,3 \%)$, seguida por Francia $(6,4 \%)$ e Italia $(6,0 \%)$. 
y Resiliencia, con 672.500 millones de euros en préstamos y subvenciones disponibles para apoyar las reformas e inversiones emprendidas por los países de la UE. El objetivo es mitigar el impacto económico y social de la pandemia de coronavirus y hacer que las economías y sociedades europeas sean más sostenibles y resilientes y estén mejor preparadas para los retos y las oportunidades de las transiciones ecológica y digital.

Por otro lado, está la Ayuda a la Recuperación para la Cohesión y los Territorios de Europa (REACT-EU), dotada con 47.500 millones de euros. Se trata de una nueva iniciativa que continúa y amplía las medidas de respuesta y reparación de crisis aplicadas a través de la Iniciativa de Inversión en Respuesta al Coronavirus y la Iniciativa de Inversión en Respuesta al Coronavirus Plus. Contribuirá igualmente a una recuperación ecológica, digital y resiliente de la economía. Los fondos se pondrán a disposición del Fondo Europeo de Desarrollo Regional (FEDER), del Fondo Social Europeo (FSE) y del Fondo de Ayuda Europea para las Personas Más Desfavorecidas (FEAD).

Por último, están las aportaciones adicionales a otros programas o fondos europeos, como Horizonte 2020, InvestEU, Desarrollo Rural o el Fondo de Transición Justa (FTJ), sumando globalmente una cuantía que ronda los $28.000 \mathrm{mi}-$ llones de euros.

Esta importante movilización de recursos implicará para España unos 150.000 millones de euros en forma de transferencias o ayudas directas y préstamos para el periodo 2021-2026 28 .

Las asignaciones que se conocen son solo las ayudas directas, que ascienden a 69.500

\footnotetext{
28 Aunque inicialmente eran 140.000 millones, se elevó la cifra en enero cuando la CE revisó cifras de impacto COVID en los países UE. Ahí fue cuando superamos a Italia.
}

millones del Mecanismo EU de Recuperación y casi 11.000 millones del REACT-EU, lo que en total suma casi 80.000 millones de euros (79.500). Esta cifra supone que España será el Estado miembro que más volumen de fondos en ayudas directas va a recibir de toda la UE. La otra parte, la relativa a préstamos, está todavía por definir, pero van a estar ligados al PIB y al plan de recuperación que se presente por cada país.

Por lo que se refiere al resto de EE MM de la $U^{29}$, en lo que respecta a ayudas directas, después de España, se situaría Italia (68.900 millones euros), seguida de Francia (39.400 millones de euros), Alemania (25.600 millones de euros) y Polonia (23.900 millones de euros). Todos ellos muy por delante del resto de países afectados.

Más allá del enorme reto que supone una gestión eficiente de dichos recursos, parece innegable que deben favorecer una transición ordenada pero decidida hacia los objetivos de sostenibilidad, digitalización y resiliencia de la economía española.

Para conseguirlo, el papel de los fondos europeos para la recuperación debe ser absolutamente esencial desde una doble perspectiva: por un lado, en la medida en que se conviertan en un elemento tractor incentivador de cambios en los comportamientos de los operadores públicos y privados; por otro, convirtiéndose en el principal mecanismo de compensación de los potenciales desequilibrios en el mercado interior provocados por las diferencias entre Estados miembros de la UE.

El 7 de octubre de 2020, el Reino de España había presentado a la CE las principales líneas estratégicas de ejecución de $\operatorname{los} \triangleright$

29 https://ec.europa.eu/info/strategy/eu-budget/long-term-eubudget/2021-2027/spending/budget-pre-allocations_en. 
fondos con el denominado Plan de Recuperación, Transformación y Resiliencia de la Economía.

Para instrumentar a nivel interno el paquete de medidas acordado por la Unión Europea, vinculándolo a los diferentes expedientes de gasto que se pueden manejar desde la óptica pública (contratación pública, subvenciones, convenios...), el 31 de diciembre de 2020 se publicó en el BOE el Real Decreto-ley 36/2020, de 30 de diciembre, por el que se aprueban medidas urgentes para la modernización de la Administración Pública y para la ejecución del Plan de Recuperación, Transformación y Resiliencia.

En cualquier caso, para llevar a la práctica dichos objetivos, y en especial con respecto al reto de la sostenibilidad, cabe preguntarse cuál puede ser el papel de la política de competencia. Abordamos esa pregunta en el siguiente apartado.

\subsection{La política de competencia y la sostenibilidad}

La política de competencia está integrada por un conjunto variado de actuaciones que van desde la defensa de la competencia (persecución de conductas anticompetitivas y control de las concentraciones económicas) a la promoción de la competencia (dentro de la cual se incluye el control sobre la actividad de los poderes públicos que afecte a la competencia) en todos los mercados y sectores productivos.

En el Marco Temporal, la Comisión Europea señalaba que diseñar medidas nacionales de apoyo de forma que cumplan los objetivos estratégicos de la UE en relación con la transformación ecológica y digital de sus economías permitirá un crecimiento más sostenible a largo plazo y fomentará la transformación hacia el objetivo acordado por la UE de alcanzar la neutralidad climática para 2050.

Por estas razones, la CE lanzó en octubre de 2020 una consulta pública en la que solicitaba contribuciones a expertos, autoridades, academia, industria y consumidores para afrontar, en su caso, cambios en el modelo existente. La Comisión Nacional de los Mercados y la Competencia (CNMC) ha contribuido a la consulta ${ }^{30}$ en su papel de autoridad de competencia y regulación.

En su contribución, la CNMC recuerda que la política de competencia no tiene entre sus objetivos primordiales la sostenibilidad, sino la eficiencia y el buen funcionamiento de los mercados, en beneficio de los consumidores. Sin embargo, la relación entre las normas de competencia y la sostenibilidad, no solo desde el punto de vista medioambiental, sino también en sus dimensiones económica y social, es una de las cuestiones más relevantes para la agenda de las autoridades de competencia en los próximos años.

En este sentido, la trascendencia de la transición hacia un modelo de desarrollo más sostenible conlleva la obligación de que todas las políticas públicas (incluida la de competencia) deban, en la medida de sus posibilidades, integrar y adaptarse a sus objetivos.

Actualmente, la política de competencia ya tiene una incidencia propia en el logro de la sostenibilidad al propiciar la innovación tecnológica y fomentar la competencia entre empresas para ofrecer al consumidor productos y servicios más sostenibles. La sostenibilidad debe ser considerada como un factor diferencial de competitividad que juega a favor de $\triangleright$

30 Puede consultarse la contribución de la CNMC a la consulta pública de la CE sobre la política de competencia y los objetivos de sostenibilidad medioambiental. 
los consumidores y que la política de competencia debe reforzar.

En cualquier caso, a pesar de lo anterior, la política de competencia no ocupa el primer puesto en la lucha contra el cambio climático y la protección del medio ambiente. Hay otras formas mucho más efectivas de hacerlo, como la regulación y la fiscalidad. Esto implica que, salvo que se produzcan cambios en el marco jurídico vigente, la contribución de la política de competencia al denominado Pacto Verde Europeo (Green Deal) ${ }^{31}$ solo puede efectuarse dentro de estos límites claramente definidos.

No obstante, la política de competencia puede complementar la regulación, y la cuestión radica en cómo podría hacerlo de la manera más eficaz. Aunque competencia y sostenibilidad son complementarias, en ocasiones se producen zonas de fricción, por lo que debe alcanzarse un equilibrio socialmente satisfactorio para ambas. Utilizar los principios de buena regulación y, en concreto, el test de necesidad y proporcionalidad es la mejor forma de determinar dicho punto de equilibrio ${ }^{32}$.

En su contribución, la CNMC señala que cualquier modulación del análisis de competencia por consideraciones de sostenibilidad debe ajustarse a cuatro principios:

- Dar predictibilidad a los operadores económicos, de manera que cuenten con la certeza necesaria sobre cómo las autoridades valorarán sus actuaciones con arreglo al derecho de competencia. De otro modo, se desincentivarán acciones que pueden mejorar en la sostenibilidad.

\footnotetext{
31 Comunicación de la CE de 11 de diciembre de 2019 COM(2019) 640 final sobre el Pacto Verde Europeo.

32 Si una actuación restringe la competencia, debe hacerlo porque sea necesario para garantizar una razón imperiosa de interés general (principio de necesidad) y de la forma menos lesiva posible (principio de proporcionalidad).
}

- Mantener la coherencia en la interpretación de las normas de competencia dentro de la UE, evitando la fragmentación del mercado interior y que los operadores puedan esperar resultados diferentes de los análisis de competencia en función de quién sea la autoridad (o el juez) que lleve a cabo el análisis.

- Mantener la competencia efectiva en el mercado interior, evitando las prácticas y operaciones empresariales restrictivas de la competencia y las distorsiones sobre la competencia en el mercado interior ocasionadas por las ayudas de Estado. Se trata de efectuar un «ajuste fino» en la política de competencia para incorporar las consideraciones de sostenibilidad medioambiental, no una transformación radical del marco legal vigente.

- Mantener la coherencia del conjunto de las normas de competencia, evitando que una mayor sensibilidad por cuestiones de sostenibilidad socave la coherencia de las normas de competencia (por ejemplo, que se cuestionen los objetivos de eficiencia y beneficio de los consumidores, tanto en el corto como en el largo plazo).

Por lo que se refiere específicamente a la utilización de las ayudas públicas como instrumento catalizador de la transición medioambiental, las recomendaciones de la CNMC se asientan en los siguientes elementos:

- Facilitar la tramitación de las ayudas medioambientales mediante la agilización de los procedimientos o mediante la revisión de las directrices de ayudas de Estado puede tener un impacto positivo sobre esta clase de ayudas $\sin \triangleright$ 
afectar significativamente a la competencia ${ }^{33}$. En particular, la CNMC considera que sería recomendable facilitar la tramitación de las ayudas al autoconsumo y a la movilidad sostenible en el sector del transporte. Igualmente, un nuevo enfoque que priorice las ayudas a consumidores presenta, a priori, un menor riesgo de distorsión de la competencia que la concesión de ayudas a grandes productores.

- El control de las ayudas de Estado tiene una naturaleza fundamentalmente económica en cuanto al análisis prospectivo de los efectos de los proyectos notificados $^{34}$. Introducir una dimensión medioambiental en la evaluación ${ }^{35}$ puede dificultar la ponderación de costes y beneficios por parte de la Comisión Europea y reducir la seguridad jurídica sobre sus decisiones.

- El control de las ayudas de Estado es un mecanismo fundamental para equilibrar a las empresas de los Estados miembros de la UE. Un control más relajado puede distorsionar la competencia en el mercado interior al favorecer la creación de campeones nacionales o fortalecer la posición de las empresas de los países con mayor capacidad financiera, que se apropiarían de una parte inequitativa de las ganancias derivadas del mercado interior. Un ejemplo de las diferentes capacidades entre Estados miembros son las

33 En particular, las Directrices sobre ayudas estatales en materia de protección del medio ambiente y energía 2014-2020.

34 Ello sin perjuicio de que el control tiene una dimensión más amplia. Por ejemplo, la Comunicación sobre la modernización de las ayudas estatales incluye como objetivo del control «impulsar un crecimiento sostenible, inteligente e integrador en un mercado interior competitivo".

35 Por ejemplo, si se requiere a la Comisión Europea la elección entre un determinado grado de perjuicios sobre la competencia y determinadas ventajas sobre el medio ambiente. diferencias entre países en los niveles de ayudas concedidas durante la pandemia de la COVID-1936. Toda apertura del control sobre esta clase de ayudas estatales que permita la existencia de una competencia entre Estados miembros debe compensarse con mecanismos para equilibrar las capacidades financieras de los mismos.

\section{Conclusiones}

La respuesta de la UE a la pandemia de la COVID-19 se ha instrumentado esencialmente a través de la aprobación de un Marco Temporal de concesión de ayudas públicas que flexibiliza las reglas del juego existentes en esta materia. Este Marco Temporal ha resultado clave para que los Estados hayan podido canalizar importantes volúmenes de ayuda a sus empresas en muy poco tiempo. Al mismo tiempo, una mayor flexibilidad con las ayudas de Estado puede implicar mayores diferencias entre Estados miembros en función de su capacidad financiera.

Si bien siempre han existido diferencias entre EE MM de la UE, en cuanto al volumen de ayuda concedida a sus empresas nacionales, en la actualidad existe un riesgo alto de desequilibrios en el mercado interior como consecuencia de las enormes diferencias de músculo financiero disponible en ellos.

Sin perjuicio del reto de gestión que supone, una manera de compensar estos desequilibrios podría ser a través de los fondos europeos de recuperación y la consecución de $D$

36 Como se ha expuesto, en el contexto actual hay enormes diferencias en la cantidad de ayudas concedidas por los diferentes Estados miembros, que están ligadas al espacio presupuestario del que disponen, así como al tamaño respectivo de sus economías. 
los objetivos horizontales de transición digital y sostenibilidad medioambiental.

Por último, nos planteamos la relación entre la política de competencia y el objetivo de sostenibilidad. Cabe recordar que la política de competencia no tiene entre sus objetivos primordiales la sostenibilidad, sino la eficiencia y el buen funcionamiento de los mercados, pero puede complementar a otros instrumentos de política pública (regulación, fiscalidad) a través de, entre otras herramientas, la aplicación de los principios de buena regulación.

\section{Bibliografía}

Comisión Nacional de los Mercados y la Competencia (2020). Contribución de la CNMC a la consulta pública de la Comisión Europea sobre la política de competencia y los objetivos de sostenibilidad medioambiental. https://www.cnmc. es/prensa/cnmc-posicion-green-deal-20201210

Comisión Nacional de los Mercados y la Competencia (2020). Informe anual de la CNMC de ayudas públicas. https://www.cnmc.es/ambitos-deactuacion/promocion-de-la-competencia/ayudaspublicas

Cuadro-Sáez, L., López-Vicente, F. S., Párraga Rodríguez, S., y Viani, F. (2020). Medidas de Política Fiscal en respuesta a la crisis sanitaria en las principales economías del área del euro, Estados Unidos y Reino Unido. Documentos ocasionales (2019). Banco de España. https://www. bde.es/f/webbde/SES/Secciones/Publicaciones/ PublicacionesSeriadas/DocumentosOcasionales/20/Fich/do2019.pdf

Orden PCM $/ 679 / 2020$, de 23 de julio, por la que se publica el Acuerdo del Consejo de Ministros de 21 de julio de 2020, por el que se establece el funcionamiento del Fondo de apoyo a la solvencia de empresas estratégicas. Boletín Oficial del Estado, n. -201 , de 24 de julio de 2020, pp. 56712 a 56722 . https://www.boe.es/boe/dias/2020/07/24/pdfs/ BOE-A-2020-8450.pdf
Presidencia de Gobierno, Gobierno de España (2020). Plan de Recuperación, Transformación y Resiliencia de la Economía. España Puede. https://www.lamoncloa.gob.es/presidente/actividades/Paginas/2020/espana-puede.aspx

Ministerio de Asuntos Económicos y Transformación Digital (2020). Medidas Adoptadas COVID-19. https://portal.mineco.gob.es/es-es/ministerio/covid19/Paginas/ART_CABECERA.aspx

Real Decreto-ley 25/2020, de 3 de julio, de medidas urgentes para apoyar la reactivación económica y el empleo. Boletín Oficial del Estado, n.․ 185, de 6 de julio de 2020, pp. 47684 a 47742 . https:// www.boe.es/eli/es/rdl/2020/07/03/25

Real Decreto-ley 36/2020, de 30 de diciembre, por el que se aprueban medidas urgentes para la modernización de la Administración Pública y para la ejecución del Plan de Recuperación, Transformación y Resiliencia. Boletín Oficial del Estado, n.․ 341, de 31 de diciembre de 2020, pp. 126733 a 126793. https://www.boe.es/boe/ dias/2020/12/31/pdfs/BOE-A-2020-17340.pdf

Real Decreto-ley 5/2021, de 12 de marzo, de medidas extraordinarias de apoyo a la solvencia empresarial en respuesta a la pandemia de la COVID-19. Boletín Oficial del Estado, n. 62 , de 13 de marzo de 2021, pp. 29126 a 29168. https:// www.boe.es/boe/dias/2021/03/13/pdfs/ BOE-A-2021-3946.pdf

Unión Europea (2014). Comunicación (2014/C 200/01) de la Comisión, de 28 de junio de 2014, de Directrices sobre ayudas estatales en materia de protección del medio ambiente y energía 2014-2020. Diario Oficial de la Unión Europea. https://eur-lex.europa.eu/legal-content/ES/ TXT/?uri=CELEX\%3A52014XC0628\%2801\%29

Unión Europea (2019). Comunicación (COM(2019) 640 final) de la Comisión, de 11 de diciembre de 2019, sobre el Pacto Verde Europeo. Diario Oficial de la Unión Europea. https://eur-lex.europa. eu/legal-content/ES/TXT/HTML/?uri=CELEX: 52019DC0640\&from $=$ EN 
Unión Europea (2020). Competition policy contributing to the European Green Deal. Comisión Europea. https://ec.europa.eu/competition/information/green_deal/index_en.html

Unión Europea (2020). Comunicación (2020/C 91 I/01) de la Comisión, de 20 de marzo de 2020, sobre el Marco Temporal relativo a las medidas de ayuda estatal destinadas a respaldar la economía en el contexto del actual brote de COVID-19. Diario Oficial de la Unión Europea. https://eur-lex.europa.eu/legal-content/ES/TXT/ $\mathrm{PDF} /$ ?uri=CELEX:52020XC0320(03)\&from=ES

Unión Europea (2020). Comunicación (2020/C 125/01) de la Comisión, de 17 de abril de 2020, sobre autorización de las ayudas estatales en el marco de las disposiciones de los artículos 107 y 108 del Tratado de Funcionamiento de la Unión Europea. Casos con respecto a los cuales la Comisión no presenta objeciones. Diario Oficial de la Unión Europea. https://eur-lex.europa.eu/legal-content/ES/TXT/PDF/?uri=OJ:C:2020: 125:FULL\&from=EN

Unión Europea (2020). Comunicación (2020/C 144/01) de la Comisión, de 30 de abril de 2020, sobre autorización de las ayudas estatales en el marco de las disposiciones de los artículos 107 y 108 del Tratado de Funcionamiento de la Unión Europea. Casos con respecto a los cuales la Comisión no presenta objeciones. Diario Oficial de la Unión Europea. https://eur-lex.europa.eu/ legal-content/ES/TXT/PDF/?uri=OJ:C:2020:144: FULL\&from $=E N$

Unión Europea (2020). Comunicación (2020/C 158/01) de la Comisión, de 8 de mayo de 2020, sobre autorización de las ayudas estatales en el marco de las disposiciones de los artículos 107 y 108 del Tratado de Funcionamiento de la Unión Europea. Casos con respecto a los cuales la Comisión no presenta objeciones. Diario Oficial de la Unión Europea. https://eur-lex.europa.eu/ legal-content/ES/TXT/PDF/?uri=OJ:C:2020:158: FULL\&from $=E N$

Unión Europea (2020). Comunicación (C(2020) 3156 final) de la Comisión, de 8 de mayo de 2020, sobre la segunda modificación del Marco
Temporal de medidas de ayuda estatal para apoyar la economía en el actual brote de COVID-19. Diario Oficial de la Unión Europea. https://ec.europa.eu/competition/state_aid/what_is_new/ sa_covid19_2nd_amendment_temporary_framework_en.pdf

Unión Europea (2020). Comunicación (2020/C 164/03) de la Comisión, de 13 de mayo de 2020, sobre la Modificación del Marco Temporal relativo a las medidas de ayuda estatal destinadas a respaldar la economía en el contexto del actual brote de COVID-19. Diario Oficial de la Unión Europea.https://www.boe.es/doue/2020/164/Z0000300015.pdf

Unión Europea (2020). Comunicación (2020/C 218/03) de la Comisión, de 2 de julio de 2020, sobre la tercera modificación del Marco Temporal relativo a las medidas de ayuda estatal destinadas a respaldar la economía en el contexto del actual brote de COVID-19. Diario Oficial de la Unión Europea. https://www.mineco.gob.es/stfls/ mineco/economia/ficheros/pdf/terceramodificacionmarcotemporalcomunitario.pdf

Unión Europea (2020). Comunicación (C(2020) 5414 final) de la Comisión, de 31 de julio de 2020, sobre casos de ayuda estatal: SA.57659 ES-COVID-19. Fondo de recapitalización. https:// ec.europa.eu/competition/state_aid/cases1/ 202032/287300_2178137_69_2.pdf

Unión Europea (2020). Comunicación (C(2020) 5504 final) de la Comisión, de 5 de agosto de 2020 sobre casos de ayuda estatal: SA.58096 COVID-19: Modificaciones a SA.56803. Esquema de garantía. https://ec.europa.eu/competition/state_aid/cases1/202032/287409_ 2179538_45_2.pdf

Unión Europea (2 de octubre de 2020). Coronavirus: Commission Statement on consulting Member States on proposal to prolong and adjust State aid Temporary Framework. Press corner. Comisión Europea. https://ec.europa.eu/commission/ presscorner/detail/en/STATEMENT_20_1805

Unión Europea (2020). Comunicación (2020/C 340 I/01) de la Comisión, de 13 de octubre de $D$ 
2020, sobre la cuarta modificación del Marco Temporal relativo a las medidas de ayuda estatal destinadas a respaldar la economía en el contexto del actual brote de COVID-19 y modificación de la Comunicación de la Comisión a los Estados miembros sobre la aplicación de los artículos 107 y 108 del Tratado de Funcionamiento de la Unión Europea al seguro de crédito a la exportación a corto plazo. Diario Oficial de la Unión Europea. https://eur-lex.europa.eu/legal-content/ES/TXT/ PDF/?uri=CELEX:52020XC1013(03)\&from=EN

Unión Europea (2020). Comunicación (2020/C 376/01) de la Comisión, de 6 de noviembre de 2020, sobre autorización de las ayudas estatales en el marco de las disposiciones de los artículos 107 y 108 del Tratado de Funcionamiento de la Unión Europea. Casos con respecto a los cuales la Comisión no presenta objeciones. Diario Oficial de la Unión Europea. https://eur-lex.europa. eu/legal-content/ES/TXT/PDF/?uri=OJ:C:2020: 376:FULL\&from=EN

Unión Europea (2020). Comunicación (2020/C 421/01) de la Comisión, de 4 de diciembre de 2020, sobre autorización de las ayudas estatales en el marco de las disposiciones de los artículos 107 y 108 del Tratado de Funcionamiento de la Unión Europea. Casos con respecto a los cuales la Comisión no presenta objeciones. Diario Oficial de la Unión Europea. https://eur-lex.europa. eu/legal-content/ES/TXT/PDF/?uri=OJ:C:2020: 421:FULL\&from=EN
Unión Europea (2020). Summary of EU Budget amount assigned to EU countries for specific programmes in the several policy areas. Budget pre-allocations. Comisión Europea. https://ec.europa.eu/info/strategy/eu-budget/long-term-eubudget/2021-2027/spending/budget-preallocations_en

Unión Europea (2021). Comunicación (2021/C 25/01) de la Comisión, de 22 de enero de 2021, sobre autorización de las ayudas estatales en el marco de las disposiciones de los artículos 107 y 108 del Tratado de Funcionamiento de la Unión Europea. Casos con respecto a los cuales la Comisión no presenta objeciones. Diario Oficial de la Unión Europea. https://eur-lex.europa.eu/legal-content/ES/TXT/PDF/?uri=OJ:C:2021:025: FULL\&from $=E N$

Unión Europea (2021). Comunicación (2021/C 34/06) de la Comisión, de 1 de febrero de 2021, sobre la quinta modificación del Marco Temporal relativo a las medidas de ayuda estatal destinadas a respaldar la economía en el contexto del actual brote de COVID-19 y modificación del anexo de la Comunicación de la Comisión a los Estados miembros sobre la aplicación de los artículos 107 y 108 del Tratado de Funcionamiento de la Unión Europea al seguro de crédito a la exportación a corto plazo. Diario Oficial de la Unión Europea. https://eur-lex.europa.eu/legal-content/ ES/TXT/PDF/?uri=CELEX:52021XC0201(01) \&from $=E S$ 